Background Many patients with life-limiting illnesses will require analgesia. Patients with complex pain symptoms are commonly prescribed regular strong opioids. A number of factors, both intrinsic and extrinsic can lead to opioid toxicity whilst this can often be managed conservatively; naloxone is sometimes required. Clinical staff who are not familiar with managing patients with palliative care needs may not be aware of the potential adverse effects if naloxone is used inappropriately. Here, we describe two cases where naloxone was used. Both of these patients had metastatic malignancies and were in-patients at an acute trust.

Aim To review and reflect on two cases where naloxone was used in patients with terminal malignancies who were receiving regular strong opioids and exhibiting signs of opioid toxicity. To compare the practice seen in the two cases against the available guidance on naloxone use in palliative care patients.

Methods Two palliative care patients were identified as receiving naloxone for opioid toxicity. Local and national guidance on the use of naloxone in palliative care was used to review the case management.

Results One case exhibits the appropriate use of naloxone, which led to a satisfactory outcome. In contrast the other case illustrates inappropriate use of naloxone, leading to severe rebound pain. This review demonstrates the importance of being able to identify when naloxone is necessary in palliative care patients without risking unnecessary reversal of analgesia. Conclusions These cases illustrate the importance of appropriate use of naloxone in palliative care patients receiving regular strong opioids. Inappropriate use of naloxone can result in severe rebound pain and opioid withdrawal. Raising awareness of local and national guidelines alongside continued education to clinical staff is vital to ensure the appropriate and safe use of naloxone in palliative care patients.

\section{P-133 HYPOMAGNESAEMIA - DOES IT EXACERBATE PAIN?}

1,2Séamus Coyle, ${ }^{3}$ Daniel Monnery. ${ }^{1}$ Willowbrook Hospice, Prescot, UK; ${ }^{2}$ MCPCIL, University of Liverpool; ${ }^{3}$ Woodlands Hospice, Liverpool, UK

\subsection{6/bmispcare-2017-hospice.158}

Background Hypomagnesaemia is a common electrolyte abnormality, in the chronic state its symptoms are insidious and often non-specific. It is often undiagnosed and thus untreated. There is evidence from animal studies to suggest that magnesium is involved in pain control including an animal model of hyperalgesia which is induced by hypomagnesaemia. However the role of magnesium as an analgesic in patients is unclear. We report two cases of patients with advanced cancer who were admitted to a hospice for pain control which improved when their hypomagnesaemia was treated.

Cases Case 1 was a 73 year old man with non small cell lung cancer with metastatic bone pain. Case 2 was a 64 year old man with large cell neuroendocrine cancer with rib wall invasion.

Case Management Both patients were found on admission to have asymptomatic hypomagnesaemia and were treated with IV magnesium. Case outcome treatment for hypomagnesaemia resulted in an improvement in pain control such that analgesia was significantly decreased.

Conclusions The incidence of hypomagnesaemia in palliative patients is unknown although it is thought to be common.
These cases suggest that treating hypomagnesaemia may improve patients' pain control.

\section{P-134 PATIENT-CENTRED QUALITY IMPROVEMENT IN PALLIATIVE AND END OF LIFE CARE: LEARNING FROM ONLINE FEEDBACK}

${ }^{1}$ Sarah Russell, ${ }^{2}$ Gina Alexander, ${ }^{3}$ James Munro. ${ }^{1}$ Hospice UK, London, UK; ${ }^{2}$ Care Opinion, Stirling, UK; ${ }^{3}$ Care Opinion, Sheffield, UK

10.1136/bmjspcare-2017-hospice. 159

Background Patient and family feedback is fundamental to care as it provides information about the quality of care experiences and involves patients and the public. Feedback methods vary e.g., face to face conversations, questionnaires, touch screens etc. However, near real-time, online feedback about care and services is under developed in palliative and end of life care (PEOLC).

We report upon a 'work in progress' quality improvement project using an online platform which enables patients/families to feedback recent PEOLC experiences, leading to learning and change in the organisation. Experience with online feedback suggests that both patients and staff feel that clinical teams should be directly involved in responding online.

Aim(s) The aims over two years are to:

1. Test the value and effectiveness of near real-time online feedback in 10 UK-wide PEOLC hospital, hospice or community teams

2. Teach and support the teams how to use the online platform and use feedback for learning, service development, change and demonstrate patient-led outcomes.

Methods(s) May 2017 to Sept 2019; 10 PEOLC clinical teams (up to 20 people per team) are being educated and supported in using online feedback in their service. This includes creating email alerts, responding, reporting, and data visualisations. The programme offers a 'community of practice' (CoP) approach to supporting staff in learning to use feedback for quality improvement and wider cultural change.

Results A mixed method evaluation using qualitative (to understand the experience) and quantitative (to measure the activity) data, from patient, team and organisation perspectives, including: online platform data, experiences, outcome measurements, staff confidence, patient/family views, QI activity, CoP interaction and turning learning into care.

Conclusion Over two years the project will provide an ongoing understanding of the challenges, opportunities and outcomes in using near-real time feedback via an online platform in PEOLC. With recommendations for implementing in other PEOLC settings.

\section{P-135 PATIENT CASE STUDIES - LEARNING FROM OUR SERVICE USERS}

Marissa Bravington-Turner. Thames Hospice, Windsor, UK

\subsection{6/bmjspcare-2017-hospice. 160}

Background and Aims An organisation that places the patient voice at the centre of its delivery, is an organisation that will be totally fit for purpose. Capturing this voice is a constant challenge to hospices given the nature of palliative care. However, it is a challenge which we are continually striving to 